\title{
X-RAY APPEARANCES IN CHRONIC RHEUMATISM BY
}

\author{
GEORGE D. STEVEN
}

From the Royal National Hospital for Rheumatic Diseases, Bath

Radiography provides us with a means of studying the structure and architecture of bones and joints. The normal bone shadowing presents definite and recognizable features. The fundamental processes which maintain the normal bone appearance throughout life are few in number, and disturbance of any of these processes is a feature of more than one disease. It is fortunate, for the purpose of differential diagnosis, that the diseases have certain individual characteristics in the combination of changes they produce when disturbing these fundamental processes. One type of change may be specially pronounced, but in that case others must be looked for before the evidence can be considered of more than presumptive diagnostic value. As one might expect in diseases of chronic type, changes at various stages of activity or quiescence may be found in one and the same patient. It is the active processes which produce the characteristic features, whereas reconstructive and degenerative changes may obscure the nature of the quiescent lesions. Gross abnormality, therefore, is often a hindrance rather than a help even to the skilled observer. It may be just as necessary to search assiduously with a magnifying lens over bones which are grossly osteoporosed or honeycombed with punched-out areas as it is for minimal changes of early disease. The clinician and the pathologist are no doubt faced with somewhat similar difficulties in the complex phases of these diseases, and no one form of examination should be considered a short cut to the diagnosis of chronic rheumatism. The experienced radiologist usually correlates his findings with the clinical and pathological features, as these must have some bearing on the interpretation of changes which appear on a radiograph. By this means he may be able to give a presumptive report-when certain signs are present, instead of an indeterminate one which would be of no informative value. To conform with this team work, the radiologist usually refers in his report to changes being of a certain disease type. This leaves the clinician to decide if further investigation is required before the diagnostic label is applied to the case.

In the following brief survey the principal diseases and disorders which will be considered are the familiar triad, rheumatoid arthritis, osteo-arthritis, and gout. Where terms such as " early", " established ", "late ", " active", or " quiescent", are used, they will refer to local lesions unless otherwise stated, since all forms may be found in one and the same patient, whether the disease is early or advanced. 


\section{Rheumatoid Arthritis}

The early changes of active disease (presumptive) are : (1) osteoporosis of generalized type

- affecting all the bones of the skeleton : exaggeration of contrast in shadowing between shafts and ends of bones ; (2) spindle-shaped swelling of soft tissues around the affected finger joints, and possibly slight increase in the joint space from effusion.

The established changes of active disease (typical) are : (1) an increase in generalized osteoporosis : local patterns from predominance of osteoporosis in the more vascular areas ; (2) diminution of cartilage of uniform character ; (3) localized osteoporosis affecting the articular cortex of some joints ; (4) erosion of articular cortex and underlying spongiosa, especially at the articular margins, with a localized osteoporosis in the eroded trabeculae.

Late changes of very chronic or quiescent disease (obscured) are : (1) return to noimal contrast between spongiosa and compact bone, but with recovery of the bone density rarely complete ; (2) the loss of cartilage maintained or increased, and remaining uniform in character ; (3) irregular new bone covering the eroded trabeculae, reconstructive changes with cystic enclosures, and greater density in the ends and shafts of bones where articular surfaces are eroded ; (4) subluxation, especially ulnar deviation of the fingers ; (5) ankylosis, especially across gliding joints destroyed by erosion ; (6) spiky lipping at the articular margins, and scanty sclerosis of secondary osteo-arthritic type.

\section{OSTEOPOROSIS}

Osteoporosis in rheumatoid arthritis is of a generalized type affecting all the bones of the skeleton. For technical reasons it is best demonstrated in the bones of the hands where its early detection is possible. The hands also serve to show a bilateral (general) involvement. Although the osteoporosis is general in character, it predominates in more vascular bone such as the spongiosa. The result of this is to exaggerate in a striking manner the normal contrast in structural shadowing between the shafts and ends of bone. The richer blood supply on either side of an epiphyseal line causes translucent zones to appear there, and a distinctive pattern is produced by this in large bones, especially in the lateral radiograph of the knee joint. This pattern is helpful in demonstrating osteoporosis if no special technique has been employed for the radiograph of the hands, and sometimes if the condition in the hands is relatively quiescent. Large bones may also show a mottled appearance when the process is very active. These patterns are only of value as manifestations of the abruptness of the process in rheumatoid arthritis. They may be seen in other diseases, but not, for example, in osteoporosis of senile type where the process is more gradual and orderly. The diminution of opacity in the spongiosa is due to the trabeculae becoming progressively thinner, but there is no actual destruction of trabeculae as in local infections. The translucent intertrabecular spacing is wider as a result of the thinning process, and not because of any change in the architectural structure such as one finds in hyperparathyroidism (osteitis fibrosa). Diminution in opacity of compact bone lags behind the spongiosa in active stages, but catches up when the disease is well established. In the quiescent stage there is a return to the normal contrast in structural shadowing, but recovery of bone density is seldom if ever complete.

The radiographic features observed in generalized osteoporosis of rheumatoid 
arthritis are consistent with pathological findings, suggesting that the process is one of deossification by osteoclastic activity. There is a disturbance of the mechanism which controls the balance between osteoclastic erosion and osteoblastic replacement in the normal living bone with the former outstripping the latter. Action on the controlling mechanism accounts for the generalized nature of the osteoporosis. As the osteoclastic process is one of cellular activity requiring oxygen and nutritional fluids, one would expect more vigorous action where there is a rich blood supply and a large surface area such as is found in the spongiosa and other areas where patterns are produced.

Generalized osteoporosis may be the only obvious radiographic sign in rheumatoid arthritis. In such a case it is consistent with the disease and of presumptive value when correlated with a rapid sedimentation rate in an adolescent or adult with spindle-shaped swellings of the finger joints. A magnifying lens should be used in the study of affected joints, and especially over the heads of the metacarpals, for the possible presence of early but significant local joint changes.

\section{Loss of Articular Cartilage}

Articular cartilage is responsible for the uniform joint space with which we are familiar. In the early rheumatoid case the space in some of the smaller joints may be slightly widened by synovial effusion, and spindle-shaped swelling may be noted in the surrounding soft tissues. In established rheumatoid arthritis the local joint changes present a more characteristic feature in the form of diminution of joint space of a striking uniform character. The metacarpo-phalangeal, proximal interphalangeal, and carpal joints are chiefly affected, but corresponding joints of the feet and the large joints may also be involved. In late rheumatoid arthritis the loss of cartilage is maintained or increased. Cartilage is a tissue of low vascularity, and shows little evidence of recovery after gross destruction.

Special mention must be made of a group of cases which show loss of cartilage in one or two of the finger joints only, and no sign of any osteoporosis. These cases are usually of the secondary rheumatoid type, the probability. being that local or focal infection has produced a transitory or minimal general disturbance which is no longer in evidence, whereas any local damage to cartilage remains.

The radiographic appearance associated with loss of cartilage is consistent with pathological findings of surface erosion of cartifîge by a pannus of granulation tissue. Cartilage has a poor blood supply of its own, and receives nourishment from the surfaces which are better provided. As granulation tissue is highly vascular, it spreads more easily over the surfaces and attacks the cartilage uniformly from that direction.

\section{ERosion of Articular Cortex}

(a) A localized type of osteoporosis may be detected by the trained eye along the articular cortex of some joints, especially in the heads of the metacarpals close to the joint margins. It may be present with or without obvious loss of cartilage. 
It is a local sign of great importance, as it is not found in diseases causing generalized osteoporosis only. Examination of films of the same patient as the disease advances will show that this is early evidence of erosion of the articular cortex of rheumatoid type. It is given prominence as a little-known sign of local joint change. It may be the only sign of local involvement, but it is equally important to find it when adjacent joints are at a late stage, and simulating gout. The radiographic appearance is consistent with the pathological finding that granulation tissue spreads under as well as over articular cartilage. Increased vascularity associated with granulation tissue produces increased osteoclastic activity in the affected cortical bone. The appearance should be confirmed with a magnifying lens.

(b) Frank erosions of rheumatoid type have a predilection for articular margins where they destroy the cortex and scoop out the underlying spongiosa. In early stages they may be small and overshadowed or concealed by other bone, unless they happen to lie at margins shown in the standard radiographs of the hands. Anterior erosions may be equally common, but they require a special oblique projection to show them up if they are not deep enough to appear as round, translucent faults in the structural shadowing. It is most important to study an erosion in profile, as it is a feature of rheumatoid arthritis that the uncovered trabeculae show a narrow zone of local osteoporosis immediately adjacent to the erosion, whereas this is not so in gout. In established rheumatoid arthritis the erosions are usually deep enough to involve margins visible in the standard radiograph, and they sometimes spread over the articular surface in some joints. In the late stage of rheumatoid arthritis a lining of new bone covers the eroded spongiosa, and the zone of trabecular osteoporosis is no longer present. The lining is at first thin and waxy, consisting of non-lamellar bone, like callus ; but later it becomes organized and lamellar. Buttressing of damaged articular surfaces may take place, with formation of enclosed cystic areas, the cysts being also lined with a thin layer of new bone. The ends and adjacent shafts of bones which have suffered destruction of their joint surfaces show a hazy uniform density which may be partly sclerosis and partly due to impaired vascularity. These bones are less affected by the generalized osteoporosis of recrudescent disease than others which suffered less in previous attacks.

The radiographic appearances are consistent with erosion by granulation tissue, this being most vigorous in its action at the vascular articular margin. The vascularity of granulation tissue also accounts for the localized zone of osteoporosis in the uncovered trabeculae immediately adjacent to the erosions, since normal osteoclastic activity would stop abruptly at the line of erosion and the adjacent trabeculae would appear normal in density. The latter type of erosion is found in gout, which is described below as chiselled out and not osteoporosed. This differential feature between rheumatoid arthritis and gout applies while the local process is in an active phase, but is obscured at a later stage of quiescence in the local condition, when new bone covers the eroded trabeculae. One is constantly 
seeing cases with multiple lesions in this difficult stage, but the skilled observer will look for, and possibly find, one local lesion which adds to the diagnostic value of the radiograph. If no active local erosion is seen, the search may be rewarded by finding areas of osteoporosis in the articular cortex such as are described in section (a) above, and this is of particular value as a more common finding in rheumatoid arthritis. If no typical changes are discovered in the local lesions, the presence of generalized osteoporosis still remains as the chief differential feature of rheumatoid arthritis.

\section{SUBLUXATION}

Ulnar deviation of the fingers at the metacarpo-phalangeal joints is a familiar deformity of rheumatoid arthritis. It is usually a feature of late stages of the disease, but occasionally marked subluxation without evidence of erosion of joint surfaces is found. In these cases abnormal laxity or marked involvement of the capsule and capsular ligaments must be presumed. The reason for deviation in the ulnar direction is not apparent. Erosions are usually more marked on the radial side of the heads of metacarpals, but it is doubtful if local joint changes could account for such frequent displacement to the ulnar side, and a neuromuscular explanation may be forthcoming. In late stages subluxation can be still more varied, and deformity may be pronounced.

\section{ANKYLOSIS}

Bony ankylosis is seen radiographically to be a frequent end-result in rheumatoid arthritis when there is complete loss of articular cartilage and erosion of opposing articular surfaces by granulation tissue. Gliding joints, such as the carpals and tarsals, are chiefly involved, but the condition may be found in other small and large joints. It is a true bony ankylosis crossing the whole joint surface, and linear trabeculae are eventually seen running in orderly fashion along the lines of force. Dense sclerosis of articular surfaces may result instead, with clinical signs of fibrous ankylosis.

\section{Secondary Osteo-ARThritic Changes}

Degenerative changes of osteo-arthritic type are a natural sequence to rheumatoid arthritis in late quiescent stages, because of abnormal stresses and strains in the disorganized joints. Osteophytic formation is at first of spiky type, and osteosclerosis scanty ; but later the changes may become more marked and may obscure the primary condition. The appearance of uniform loss of cartilage in an apparently osteo-arthritic hip joint may be a distinguishing feature which further investigation may confirm.

\section{Osteo-arthritis}

The earliest change (presumptive) is osteophytic formation of beak-shaped type at the place of insertion of capsular ligaments to joint margins. 
Established changes (typical) are : (1) loss of cartilage of a one-sided character, resulting in faults in bone position or alignment and corresponding shift in lines of stress ; (2) osteophytic formation at margins where stress is increased, with either imperfect attempts at bone bridging or outgrowths in the direction of the plane of the joints ; (3) separate ossicles in the middle of capsular ligaments in some interphalangeal joints ; (4) hypertrophic bone outgrowths of terminal phalanges (Heberden's nodes) ; (5) osteosclerosis in subarticular bone, and coarsening of trabeculae along lines of stress.

Late changes (obscured) are : (1) gross loss of cartilage ; (2) eburnation, with pitting of articular surfaces ; (3) subarticular cystic degeneration ; (4) subluxation.

\section{OSTEOPHYTIC FORMATION}

Lipping seen at the attachments of capsular ligaments is only presumptive evidence of osteo-arthritic disease, as this is the normal reaction to excessive local stress and strain and is common in athletic subjects after middle age. It may be an incidental finding in clinical fibrositis, or evidence of old trauma. Early lipping consists of small pointed spurs or rounded knobs of uniform density which project from bone immediately adjacent to an articular margin in the region of the attachment of capsular ligaments. Because of their uniform density they do not obscure the old outline of the bone to which they are attached. As the osteophytes grow in size they become more organized in character and present at least an outer layer of compact bone. The direction in which they grow appears to vary according to the nature of the strain which stimulates their formation. When the joint surfaces are tilted from loss of cartilage on one side, the osteophytes grow out on the side of angulation or concavity and are directed in the plane of the: joint surfaces, as if to compensate for the extra strain which now falls on that side. These osteophytes usually remain pointed and may be large and very dense. When there is no gross loss of cartilage and the strain is mainly in the capsular ligaments, the osteophytes usually run in the direction of the ligaments and become beak- or hook-shaped. They are usually rounded at their ends, and may grow to large size, but they do not as a rule fuse with osteophytes which grow towards them from the opposing articular surfaces. They are not usually as dense as the pointed type, and often form part of a fringe of new bone which is more extensive than the radiographic appearance might suggest. Both types are well demonstrated in the lumbar spine, the one with wedge-shaped intervertebral disc, and the other with scoliosis from rotation of vertebral bodies. In both cases they predominate on the side of concavity. In late stages, when osteophytes are large and organized, they may show profuse outgrowths of organized bone close to the joint margin; and fragmented osteophytes or a moderate number of loose bodies may be present.

Separate ossicles of a different type are seen in the middle of the capsular ligaments of interphalangeal joints, lying opposite the joint, and sometimes in the anterior common ligament of the cervical or dorsal spine. They may be small and compact, or larger and cancellous in structure. They are characteristic of osteo-arthritic disease. Another change characteristic of osteo-arthritis is occasionally seen in the form of hypertrophic outgrowths of normal structural appearance at articular margins of terminal phalanges. They are quite different in 
appearance from spurs of compact bone, and are a distinctive feature of Heberden's nodes. The other main features of Heberden's nodes are : gross loss of cartilage ; eburnation, with pitting of articular surfaces ; subluxation, with tilt of the phalanx ; and, of course, nodules or soft-tissue swellings over the hypertrophied bone. In late quiescent stages of any disease which disorganizes and deforms a joint, the occurrence of secondary osteo-arthritis is a natural sequel to the abnormal stresses and strains across the faulty joint, and may eventually obscure the primary disease. The radiographic appearances in osteo-arthritis are consistent with pathological findings that osteoblastic activity is stimulated by stresses, strains; and pressure. Early lipping consists of bone of uniform density, like callus, and this gradually becomes larger and more organized in character. If subject to pressure, it is likely to become very dense in structure ; if to torsional strains, it may form a fringe-like outgrowth of moderately dense new bone. Osteophytes predominate on the side to which there is any abnormal shift of stress and strain, and tend to compensate for deformity in position and alignment, or to limit the movements of the joints concerned. Hypertrophic outgrowths, described as typical of Heberden's nodes, are sometimes simulated by subperiosteal outgrowths in articular tophi of gouty arthritis.

\section{Loss OF CARTilage}

Loss of cartilage is of a one-sided character, very different from rheumatoid arthritis. It occurs at the site of maximum stress and strain, and is usually more pronounced in large weight-bearing joints. The adjacent articular surfaces approximate to each other, so that joint surfaces are angled and the position or alignment of bones is altered. In late stages destruction may be almost complete, but seldom uniform. The appearance is consistent with a degenerative process in cartilage, which is a tissue of poor vascularity and low recuperative power. In osteo-arthritic disease the degenerative changes are multiarticular. Severe trauma followed by ordinary stress and strains may result in a more localized post-traumatic osteo-arthritis.

\section{OSTEOSCLEROSIS AND EBURNATION}

Osteosclerosis is seen in established disease. There is an increase in thickness of the articular cortex where loss of cartilage is present, and a process of coarsening is seen in the adjacent trabecular shadowing. Coarsening of trabeculae is prominent along new lines of force which result from faulty position or alignment, the shift being to the side of approximated articular surfaces. Contact of bone surfaces in late stages results in dense bone formation or eburnation, and pitting of the eburnated surface is not uncommon. These appearances are consistent with stimulation of osteoblastic activity by stresses and strains or pressure. The coarse trabeculae along new lines of force are more orderly and purposeful than the more imperfect attempts seen in other diseases, such as Paget's disease. 


\section{Cystic Degenkration}

Subarticular translucent areas are occasionally seen in early stages of osteoarthritis before there is any obvious loss of cartilage or osteophytic formation. These cases are probably of traumatic type. Cystic degeneration is more often a feature of late stages, particularly in large weight-bearing joints, such as the hips. The translucent areas are subarticular and may involve a portion of the articular surface. They show a wall of compact bone, varying in thickness according to the size of the joint concerned. They lie in the position of greatest stress, such as under the highest point of the acetabulum in the hip joint (rheumatoid erosions predominate in vascular areas at articular margins). In late stages they may be more numerous and endosteal. Cystic degeneration, and the formation of pseudo-cysts, may be the result of a localized inflammatory reaction due to trauma, with resulting localized osteoclastic activity. They may, on the other hand, result from small areas of aseptic necrosis.

\section{Chronic Gout}

Early changes (presumptive) are : (1) hazy soft-tissue shadowing affecting bone detail and contrast (out-of-focus appearance) ; (2) local soft-tissue swellings at joints (not fusiform).

Established changes (typical) are : (1) osseous tophi-subchondral, endosteal, and medullary -and predilection for the articular margin, with erosion of the articular cortex ; (2) loss of cartilage, formation of articular tophi; and subperiosteal outgrowths at margins of tophi ; (3) osteosclerosis, usually wavy and irregular, and irregular calcium deposits in bone.

Late changes (obscured) are : (1) osseous tophi walled in by compact bone. Coarse wavy buttressing with pinhead and honeycombed translucencies; (2) secondary osteo-arthritic changes; (3) ankylosis in some joints.

\section{Soft-tissue Changes}

Soft-tissue changes in chronic gout consist of local periarticular swellings which are not fusiform as seen in rheumatoid arthritis. The swellings and tissue changes produce a hazy shadowing which makes the structural bone detail look " out-of-focus". There is also a false impression of exaggerated contrast in the ends and shafts of the bones of the hands, almost simulating rheumatoid arthritis; but closer examination shows that there is no generalized osteoporosis in gout. Calcification of tophaceous swellings in soft tissues is seen in late stages of the local condition.

\section{OSSEOUS TOPHI}

Osseous tophi have a predilection for subchondral articular margins, and they commonly erode the adjacent articular cortex. These erosions present a typical appearance during the active process of deposition of sodium biurate crystals, provided the erosions are seen in profile. The appearance is that of complete loss of a small portion of cortex and underlying spongiosa, and two features are noted : (a) the cortex immediately adjacent is quite unaffected in its structure and density and ends abruptly at the erosion, or may even over-ride the 
translucent area ; $(b)$ the uncovered trabeculae are also of normal structure and density and not osteoporosed as in an active rheumatoid erosion. This abrupt transition from translucency to normal structural shadowing may also be detected during active deposition in endosteal tophi in the spongiosa. When small, the tophi are usually circular or oval, and are commonly seen in this form in the heads of the metatarsals. With increased deposit of sodium biurate a group of small translucencies may appear close to the periphery of the larger tophus, and if these merge together the larger tophus will show a crenated or honeycombed outline. Multiple small deposits are sometimes seen under the articular cortex, giving an appearance of spotted or zonal translucency under a cortex of normal density. In the base of a phalanx the tophi are often wedge- or lozenge-shaped with a point directed up the shaft between eroded main trabeculae. Endosteal tophi close to the medulla are obscured by overlying bone, and in the earlier stages give an impression of local osteoporosis only. During quiescence in the local process, the translucent areas become lined with new bone, which may be thin or thick according to the size of the tophus. If only a few small tophi are seen and they are lined in this way, there is difficulty in differentiating them from traumatic and other lined cysts, and more active lesions have to be looked for.

The radiographic appearances are consistent with the normal chiselling-out effect produced by osteoclasts. The process is seen to end abruptly at the margin of the eroding cells. In gout there appears to be no overactivity of the osteoclasts, but the presence of sodium biurate deposits inhibits the action of osteoblasts which would normally replace the eroded bone. In rheumatoid arthritis the erosion by osteoclasts is stimulated by granulation tissue, and the process outpaces the osteoblasts with the result that a zone of osteoporosis is seen.

The process of lining erosions of the cortex and spongiosa with new bone must be preceded by the formation of osteoid tissue which is translucent to $x$ rays. Non-lamellar bone of callus-like appearance then forms, and this is finally replaced by lamellar bone. The biurate deposits in the tophus are probably superseded by inactive material before these changes take place, and to all intents and purposes the primary condition in the local lesion becomes masked by a process common to other forms of erosion. Difficulties such as this emphasize the need for careful

- scrutinizing of local lesions and for correlation of the appearances with other changes or combination of changes. Mistakes will be avoided if this routine is followed.

\section{Loss of Cartilage AND Formation of Articular TOPHI}

Diminution of cartilage of somewhat uniform character is seen in chronic gouty arthritis. The process is not as a rule early, and is usually preceded by tophaceous deposits in the bones. In late stages the loss of cartilage may be complete, and associated with gross erosion of articular surfaces. The articular surfaces may come in contact, and the local condition may end in ankylosis.

The radiographic appearance is consistent with the finding of spiky sodium 
biurate crystals in the surface layers of articular cartilage where they have been deposited from overloaded synovial fluid. Ankylosis would be explained by a final invasion of granulation tissue.

The formation of articular tophi presents a still more typical appearance of gouty arthritis. In this case the deposit of crystals accumulates, and the eroded articular surfaces are seen to be widely separated by a translucent space, with one bone apparently floating above the other. In some of these cases another characteristic feature is seen in the form of outgrowths of subperiosteal bone from the articular margins, which go partly round the tophi, but usually end in a point as if failing in the attempt.

\section{OSTEOSClEROSIS AND BUTTRESSING}

Coarse, irregular trabecular shadowing is seen in late stages of local gouty changes in bone. The sclerosis is wavy, and not orderly as in osteo-arthritis. Denser shadowing from calcium deposits in bone may also be seen. When tophi are surrounded by sclerosis and a wall of new bone, they are quiescent, and the biurate crystals have probably been replaced by inactive material. A honeycombed appearance is frequently seen, with intercommunication between the various sized translucencies-some of them appearing like pinholes in the new bone. Buttressing of damaged joints is also seen, the structural appearance again tending to be wavy.

\section{SeCONDARY Osteo-ARTHRITIS}

Changes of secondary osteo-arthritic type appear in late stages of gouty arthritis, as may be expected in any disorganized joint which becomes liable to abnormal stresses and strains. Cancellous outgrowths are often seen at articular margins in gout, in addition to the more common spurs of compact bone. Secondary osteo-arthritis may be pronounced in large weight-bearing joints. In these joints gouty deposits are uncommon, and the primary condition may be obscured.

\section{Summary of Differential Features}

The differential features are summarized in the Table on page 11.

\section{Differential Features in Other Diseases}

ANKYLOSING SPONDYLITIS.-In ankylosing spondylitis the predominant process is bony ankylosis spreading across the joint margins along the outer edge of the articular cartilage. It is usually seen first in the sacro-iliac joints as a bilateral process somewhat similar to the normal fusion which takes place between segments of the sacrum. The normal sharp outline of the articular margins disappears, the bone immediately adjacent to the articular surfaces becomes hazy and sclerosed, and the abnormal sclerosed bone forms a new outline which is extremely ragged-so much so that one gets the impression of lacunar areas of absorption in the articular cortex which add to the irregular shadowing produced by the new articular margins. In time the space between the articular edges appears to be crossed by interlacing shadows of new bone, and at a still later stage by definite trabeculation which is arranged in orderly manner along lines of force. The extent of osteosclerosis and osteoporosis in the affected bones varies in their relative 


\begin{tabular}{|c|c|c|c|c|}
\hline & & Rheumatoid & Osteo-arthritis & Gout \\
\hline 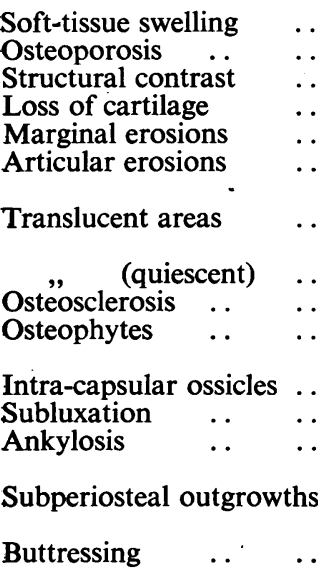 & $\begin{array}{l}\ldots \\
\ldots \\
\ldots \\
\ldots \\
\cdots \\
\because . \\
\ldots \\
\ldots \\
\ldots \\
\ldots \\
\ldots \\
. .\end{array}$ & $\begin{array}{l}\text { fusiform } \\
\text { generalized } \\
\text { exaggerated } \\
\text { uniform } \\
\text { osteoporosed } \\
\text { preceded by osteo- } \\
\text { porosis } \\
\text { trabeculae } \\
\text { osteoporosed } \\
\text { compact wall } \\
\text { scanty secondary } \\
\text { small spiky } \\
\text { secondary } \\
\text { nil } \\
\text { ulnar deviation } \\
\text { bony } \\
\text { nil } \\
\text { orderly }\end{array}$ & $\begin{array}{l}\text { nodular } \\
\text { nil } \\
\text { normal } \\
\text { one-sided } \\
\text { nil } \\
\text { pitted or pseudo- } \\
\quad \text { cystic } \\
\text { compact wall } \\
\text { compact wall } \\
\text { orderly } \\
\text { spur or fringe } \\
\text { interphalangeal } \\
\text { tilt or slip } \\
\text { incomplete } \\
\quad \text { bridging } \\
\text { at Heberden's } \\
\text { nodes } \\
\text { rare }\end{array}$ & $\begin{array}{l}\text { lumpy } \\
\text { local } \\
\text { hazy normal } \\
\text { varied } \\
\text { chiselled out } \\
\text { chiselled out } \\
\text { trabeculae } \\
\text { chiselled out } \\
\text { compact wall } \\
\text { wavy } \\
\text { hypertrophic and } \\
\text { secondary } \\
\text { nil } \\
\text { varied } \\
\text { bony } \\
\text { at articular } \\
\text { tophi } \\
\text { wavy honeycombed }\end{array}$ \\
\hline
\end{tabular}

amounts according to the acuteness of the disease. In acute cases the osteoporosis becomes very prominent and widespread, whereas in chronic cases the osteosclerosis is the more marked feature. The disease spreads centrifugally up the spine and sometimes to the symphysis pubis, and down to the joints of the lower limbs. A shaggy appearance is occasionally seen along the lower ramus of the pubic bones and ischium as a result of spiky periosteal outgrowths at tendon or ligamentous attachments. In the spine the disease involves the joints between the vertebral bodies and the articular processes, together with extension of the process of ossification to the interosseous ligaments. The ankylosing process is well shown in the lumbar and cervical spine, the lumbar spine being the most common site after the sacro-iliac joints. A uniform linear opacity is seen crossing from edge to edge of the joint between vertebral bodies along the outer aspect of the intervertebral discs (i.e. well inside the line followed by osseous bridging in spondylitis osteo-arthritica). As new bone is laid down the linear shadow broadens and may bulge out between the vertebrae so that the spine becomes bamboo-shaped. The new bone is quite dense compared with the vertebral bodies, which are usually osteoporosed. The involvement on one or other side and at different levels is usually more varied than the old text-book illustration of bamboo-spine would suggest. Involvement of interosseous and interspinous ligaments results in the so-called " tram-line" appearance, with three lines of relatively dense new bone running up the spine. Eventually these may show very strong linear trabeculation of orderly type, whereas the vertebral bodies remain osteoporosed. As regards the articular cartilage, this is enclosed and not invaded by the process, but it may eventually deteriorate. Changes in the articular processes of the spine are best seen in oblique views. A shaggy articular outline with an underlying narrow zone of dense bone may be seen in this projection. If such a combination of changes is found, the diagnosis is almost a certainty. The dorsal spine is usually less involved than the lumbar region. In some cases there is a general dorsal kyphosis with approximation of the anterior margins of the vertebral bodies, which may ankylose. The cervical spine shows ankylosis between vertebral bodies or articular processes, and not infrequently in both places. The extent to which the disease spreads varies considerably, and some cases show evidence of quiescent periods with mixed osteo-arthritic changes. The standard radiograph of the hands presents a normal appearance in all but very advanced cases, and this helps to differentiate ankylosing spondylitis from a rheumatoid arthritis which involves the spine. 
STILL's DiseaSE.-Still's disease shows the $x$-ray features of rheumatoid arthritis in children, with predominance of generalized osteoporosis. Local joint changes may supervene.

SENILE ATROPHY AND DisUSE ATROPHY.-In senile atrophy, osteoporosis is of generalized type, but the process is extremely slow and does not produce exaggerated contrast in shadowing between shafts and ends of bone. The general architecture is little disturbed. Trabeculae become thinner, and some may gradually disappear. The compact bone forms a thin, shell-like outer covering, which is present also in the articular outline (distinct from rheumatoid arthritis). The appearance of osteoporosis of disuse is somewhat similar if no infective process is involved, but the condition is confined to the affected limb.

HYPERTHYROIDISM AND HYPERVITAMINOSIS D.-These cause a more abrupt type of generalized osteoporosis, but without the local joint changes of rheumatoid arthritis.

RiCKETS AND OsteOMALACIA.-These conditions produce a generalized osteoporosis and bone softening, not from overactivity of osteoclasts but from failure of osteoid tissue to calcify. In the growing bones of children the result of this is most marked at growing ends. Deformities from bone softening are likely to occur (bowing). Expansion of intervertebral discs at the expense of softened vertebral bodies in the adult spine produces a fish-spine appearance.

HYPERPARATHYROIDISM (OSTEITIS FIBROSA).-Generalized osteoporosis may occur, but the trabecular thinning is associated with a hyperplasia of intertrabecular tissue, with the result that there is a true widening of the intertrabecular spaces which may become cystic in places. Further architectural disturbance is seen by similar spaces occurring in layers of compact bone of the shaft which appears broken up by lines of translucency.

Paget's Disease.-Widening of intertrabecular spacing is seen which is somewhat similar to osteitis fibrosa but is accompanied by marked coarsening of trabecular shadowing. The architecture is more irregular and less purposeful than normal bone, and, although strong trabeculae appear along lines of force, they are imperfectly laid down. While dense in appearance, the affected bone is diminished in strength, and bowing deformity is seen with buttressing on the concave side from subperiosteal new bone. Osteo-arthritic changes are common in affected joints. Changes of Paget type may affect only part of one or more bones.

Charcot's Disease.-Charcot's disease shows gross changes with loss of cartilage, eburnation, sclerosis, and multiple bone fragmentation.

SPECIFIC Disease.-There is irregular flaky coarse sclerosis which is not predominantly subarticular. There is also evidence of chronic periostitis, bowing, with new bone on the convex side, and local mottled shadowing or local osteoporosis.

TUBERCLE.-There is local bone or joint invasion, and local osteoporosis with ground-glass appearance in affected area (probably from the occluding effect of the tubercle on neighbouring blood vessels diminishing the osteoclastic activity compared with the surrounding vascular bone). Local trabecular destruction supervenes. In caries sicca of the shoulder joint, there is osteoporosis with articular erosions and subarticular sclerosis. The condition is usually monarticular.

Osteitis Tuberculosa Multiplex Cystica (Boeck's SARCoID).-Multiple translucent areas appear indistinguishable from quiescent gouty tophi in bones of the hands, but there is not the same predilection for marginal subchondral areas.

Carcinomatosis.- In carcinomatosis from primary carcinoma of the prostate, osteoblastic changes may obscure the destructive process. The sclerosis is flaky, but in some cases a uniform density is produced in some of the bones. Some areas of bone destruction are likely to be seen.

MYELOMATOSIS.-Irregular osteoporosis results from widespread invasion of bone structure by small cell masses. In some places round or oval areas of bone destruction are likely to be seen.

MULTIPLE ChONDROMATA AND ENCHONDROMATA.-There are multiple translucent areas with a thin covering of compact bone. These may project or expand beyond the bone surface while still retaining their compact bone covering.

TRAumatic CySTS.-These are small endosteal cysts with compact walls ; they are similar to the quiescent cysts of other conditions described. 
OSTEOCHONDRITIS.-Osteochondritis may be primary to more obvious secondary osteoarthritis. Expansion of the head of the femur, expansion and deformity of the head of the second metatarsal, and compression with sclerosis of the navicular or semilunar bones are examples.

Slipped Epiphysis : Shallow Acetabulum.-These are examples of other defects in joints which may be obscured by secondary osteo-arthritic changes.

UNUNITED os ACETABULI.-This may be mistaken for a fragmented osteophyte.

Protrusio ACETABUli.-This may be overlooked, owing to the dense osteosclerosis and secondary osteo-arthritis which obscures the fact that there has been a primary condition of bone softening.

\section{Technique}

Radiographic interpretation and differential diagnosis in chronic rheumatic diseases may be materially affected by a decision that osteoporosis is present or absent. There are certain fundamental principles in radiography which must be appreciated and applied before one can attempt to diagnose the presence of early osteoporosis. Radiographs taken of one and the same bone with different techniques will vary considerably in appearance, both as regards the detail and density of the shadows and the contrast between different parts of the bone. $X$ rays of high penetrating value give a more uniform density to bone shadows than "soft" rays which produce greater contrast between the structural components. Under-exposure and under-development give less detail and an impression of greater bone density than over-exposure or over-development, which produce the opposite effect. Different distances of the $x$-ray tube from the film have not only a pronounced effect on exposure factors, but may alter the size of the bone shadow and its structure. It is desirable that all such factors should remain constant in any standardized technique if one is to become familiar with the normal and detect the early abnormal signs. The use of constant factors is possible only if the part to be radiographed is always of the same thickness, and the only bones and joints which conform to a reasonable extent with this requirement are those of the hands. The standard radiograph of the hands has been employed for this reason by hospitals which specialize in chronic rheumatic diseases, and this radiograph is usually taken for diagnostic purposes, whether the hand is requested or not. A normal hand would be helpful as a control, but this is impracticable. A useful substitute may be employed in the form of a small wax block in which is embedded the head with part of the shaft of a metacarpal bone (preferably the second metacarpal bone set in the anterior position). The block is $2 \mathrm{~cm}$. thick, to correspond with the thickness of the hand. Wax has the same absorptive effect on $x$ rays as the soft tissues of the body. General hospitals with clinics for chronic rheumatic diseases would be well advised to employ a standard technique for radiographing hands. There are difficulties in establishing any universal standard which would permit true comparison of films from different hospitals, but these difficulties are by no means insurmountable.

In cases of ankylosing spondylitis, where the hands as a rule are normal in appearance, it is desirable that a modified standard technique be employed-at least for the pelvis and lumbar spine. This can be achieved by measuring the actual thickness of the part under examination with calipers, and employing a specially prepared graph on which thickness appears on the abscissa, and one variable exposure factor (e.g. Kv or MaS on the ordinate).

\section{Summary}

The radiographic appearances of rheumatoid arthritis, osteo-arthritis, and gout are described. Differential features of some other diseases are given briefly.

A standard radiograph of the hands is recommended as a general routine for diagnostic purposes.

Attention is drawn to differential features of the local bone and joint changes in the hands during active phases of the three diseases mentioned, and similarity 
in appearances during quiescence of the local bone condition is illustrated and explained.

Special attention is drawn to a local osteoporosis of the articular cortex which precedes and accompanies erosions of rheumatoid arthritis. It is not found in erosions from sodium biurate deposits of gouty arthritis, and a group of diseases which might simulate the generalized osteoporosis of rheumatoid arthritis do not show this local change.

It is stressed that changes in the active stages are of diagnostic value. Changes in the later stages may be predominant and misleading.

Team work with the clinician and pathologist is strongly recommended, and correlation of the radiographic interpretation with the clinical and pathological findings is advised as a routine procedure.

(For Illustrations to this Article see page 27) 